\title{
EVALUATION OF A TRIGONOMETRIC INTEGRAL
}

\section{R. MOHANTY}

1. Introduction. It is assumed throughout the present paper that $f(t) \in L(-\infty, \infty)$. Then the Fourier integral of $f(t)$ at $t=x$ is

$$
\frac{1}{\pi} \int_{0}^{\infty} d u \int_{-\infty}^{\infty} f(t) \cos u(x-t) d t
$$

This may be written in a form analogous to that of the Fourier series as

$$
\int_{0}^{\infty}\{a(u) \cos x u+b(u) \sin x u\} d u
$$

where

$$
a(u)=\frac{1}{\pi} \int_{-\infty}^{\infty} f(t) \cos u t d t, \quad b(u)=\frac{1}{\pi} \int_{-\infty}^{\infty} f(t) \sin u t d t .
$$

The allied integral of the Fourier integral is then

$$
\begin{aligned}
\int_{0}^{\infty}\{b(u) \cos x u-a(u) \sin x u\} d u & \\
= & \frac{1}{\pi} \int_{0}^{\infty} d u \int_{-\infty}^{\infty} f(t) \sin u(t-x) d t .
\end{aligned}
$$

Thus the allied integral of an odd function $f(t)$ belonging to $L(0, \infty)$ is

$$
\frac{2}{\pi} \int_{0}^{\infty} \cos x u d u \int_{0}^{\infty} f(t) \sin u t d t
$$

We write

$$
\psi(t)=f(x+t)-f(x-t) .
$$

Definition. We say that the integral $\int_{0}^{\infty} g(u) d u$ is summable $(C, 1)$ to $\operatorname{sum} I$, if

$$
\lim _{\lambda \rightarrow \infty} \int_{0}^{\lambda}\left(1-\frac{u}{\lambda}\right) g(u) d u=I .
$$

In this paper we are concerned with the integrals of the type

Received by the editors December 19, 1955 . 


$$
\int_{0}^{\infty} u^{\alpha}\{b(u) \cos x u-a(u) \sin x u\} d u,
$$

where $0<\alpha<1$, and first prove the following

Theorem. Integral $(7)$ is summable $(C, 1)$ to

$$
\frac{\Gamma(1+\alpha)}{\pi} \cos \frac{1}{2} \alpha \pi \int_{\rightarrow 0}^{\infty} \frac{\psi(t)}{t^{1+\alpha}} d t,
$$

whenever this integral exists, and

$$
\psi(t)=o\left(t^{\alpha}\right) \text { as } t \rightarrow 0 .
$$

2. Proof of the theorem. We are to consider the behaviour of

$$
\begin{aligned}
I & =\frac{1}{\pi} \int_{0}^{\lambda}\left(1-\frac{u}{\lambda}\right) u^{\alpha} d u \int_{-\infty}^{\infty} f(t) \sin u(t-x) d t \\
& =\frac{1}{\pi} \int_{0}^{\lambda}\left(1-\frac{u}{\lambda}\right) u^{\alpha} d u \int_{0}^{\infty} \psi(t) \sin u t d t
\end{aligned}
$$

as $\lambda \rightarrow \infty$. Now

$$
\int_{0}^{\lambda}\left(1-\frac{u}{\lambda}\right) u^{\alpha} d u \int_{0}^{\infty} \psi(t) \sin u t d t=\int_{0}^{\infty} \psi(t) K(\lambda, t) d t
$$

where

$$
\begin{aligned}
K(\lambda, t)= & \int_{0}^{\lambda}\left(1-\frac{u}{\lambda}\right) u^{\alpha} \sin u t d u \\
= & \lambda^{1+\alpha} \int_{0}^{1}\left(\omega^{\alpha}-\omega^{\alpha+1}\right) \sin \omega \lambda t d \omega \\
= & \lambda^{1+\alpha}\left[\left(\omega^{\alpha}-\omega^{\alpha+1}\right) \frac{\cos \omega \lambda t]^{1}}{-\lambda t}\right]_{0}^{1} \\
& +\frac{\lambda^{\alpha}}{t} \int_{0}^{1}\left[\alpha \omega^{\alpha-1}-(\alpha+1) \omega^{\alpha}\right] \cos \omega \lambda t d \omega \\
= & \frac{\alpha \lambda^{\alpha}}{t} \int_{0}^{1} \frac{\cos \omega \lambda t}{\omega^{1-\alpha}} d \omega-\frac{(\alpha+1) \lambda^{\alpha}}{t} \int_{0}^{1} \omega^{\alpha} \cos \omega \lambda t d \omega \\
= & \frac{\alpha}{t^{1+\alpha}} \int_{0}^{\lambda t} \frac{\cos \theta}{\theta^{1-\alpha}} d \theta+O\left(\frac{1}{\lambda^{1-\alpha} t^{2}}\right) .
\end{aligned}
$$

Hence 


$$
K(\lambda, t)-\frac{\alpha}{t^{1+\alpha}} \int_{0}^{\infty} \frac{\cos \theta}{\theta^{1-\alpha}} d \theta=\frac{-\alpha}{t^{1+\alpha}} \int_{\lambda t}^{\infty} \frac{\cos \theta}{\theta^{1-\alpha}} d \theta+O\left(\frac{1}{\lambda^{1-\alpha} t^{2}}\right) .
$$

By the second mean value theorem, we have

$$
\left|\int_{\lambda t}^{\infty} \frac{\cos \theta}{\theta^{1-\alpha}} d \theta\right| \leqq \frac{2}{\lambda^{1-\alpha} t^{1-\alpha}},
$$

and consequently

$$
\left|K(\lambda, t)-\frac{\Gamma(\alpha+1)}{t^{1+\alpha}} \cos \frac{1}{2} \alpha \pi\right|<\frac{A}{\lambda^{1-\alpha} t^{2}} .
$$

Plainly

$$
|K(\lambda, t)|<A \lambda^{1+\alpha} .
$$

It follows at once that, if $\delta>0$,

$$
\lim _{\lambda \rightarrow \infty} \frac{1}{\pi} \int_{\delta}^{\infty} \psi(t) K(\lambda, t) d t=\frac{\Gamma(1+\alpha)}{\pi} \cos \frac{1}{2} \alpha \pi \int_{\delta}^{\infty} \frac{\psi(t)}{t^{1+\alpha}} d t .
$$

Also by (9), we have

$$
\begin{aligned}
& \frac{1}{\pi} \int_{\lambda^{-1}}^{\delta} \psi(t) K(\lambda, t) d t \\
& \quad=\frac{\Gamma(1+\alpha)}{\pi} \cos \frac{1}{2} \alpha \pi \int_{\lambda^{-1}}^{\delta} \frac{\psi(t)}{t^{1+\alpha}} d t+O\left\{\int_{\lambda^{-1}}^{\delta} \frac{|\psi(t)|}{t^{2} \lambda^{1-\alpha}} d t\right\}
\end{aligned}
$$

and, if condition (8) holds, the last term is

$$
o\left(\frac{1}{\lambda^{1-\alpha}} \int_{\lambda^{-1}}^{\delta} t^{\alpha-2} d t\right)=o(1)
$$

by choosing first $\delta$ then $\lambda$.

Finally, by (8) and (10), we have

$$
\begin{aligned}
\left|\frac{1}{\pi} \int_{0}^{\lambda^{-1}} \psi(t) K(\lambda, t) d t\right| & <A \lambda^{1+\alpha} \int_{0}^{\lambda^{-1}}|\psi(t)| d t \\
& =o(1) .
\end{aligned}
$$

This proves the theorem.

3. Evaluation of a definite integral. Let us consider the odd function $e^{-t} t^{\beta-1}(\beta>1+\alpha)$ defined in $(0, \infty)$, then the integral $(7)$ at $x=0$ reduces for the present function to 


$$
\begin{aligned}
& \frac{2}{\pi} \int_{0}^{\infty} u^{\alpha} d u \int_{0}^{\infty} e^{-t t^{\beta-1}} \cdot \sin u t d t \\
& \quad=\frac{2}{\pi} \Gamma(\beta) \int_{0}^{\infty} \frac{u^{\alpha} \sin \left\{\beta \tan ^{-1} u\right\} d u}{\left(1+u^{2}\right)^{\beta / 2}}, \text { where } \beta>1+\alpha .
\end{aligned}
$$

Obviously this integral is a convergent integral, and since this special function satisfies the conditions of the theorem, we have by the theorem

(11) $\int_{0}^{\infty} \frac{u^{\alpha} \sin \left(\beta \tan ^{-1} u\right)}{\left(u^{2}+1\right)^{\beta / 2}} d u=\frac{\Gamma(\alpha+1) \Gamma(\beta-\alpha-1)}{\Gamma(\beta)} \cos \frac{1}{2} \alpha \pi$,

where

$$
\beta>\alpha+1 .
$$

Ravenshaw College, Cuttack, India 